\title{
Modeling Very-High-Energy Emission from Pulsars
}

\author{
Alice K. Harding, ${ }^{a, *}$ Christo Venter $^{b}$ and Constantinos Kalapotharakos ${ }^{c}$ \\ ${ }^{a}$ Los Alamos National Laboratory, Theoretical Division, \\ Los Alamos, NM, USA \\ ${ }^{b}$ North-West University, Private Bag X6001, \\ Potchefstroom 2520, South Africa \\ ${ }^{c}$ University of Maryland, College Park (UMDCP/CRESST), \\ College Park, MD, USA 20742 \\ E-mail: ahardingx@yahoo.com, christo.venter@nwu.ac.za, \\ c.kalapotharakos@gmail.com
}

Ground-based Air-Cherenkov telescopes have detected pulsations at energies above $50 \mathrm{GeV}$ from a growing number of Fermi pulsars. These include the Crab, Vela, PSR B1706-44 and Geminga pulsar, with the first two having pulsed detections above $1 \mathrm{TeV}$. In some cases, there appears to be very-high-energy (VHE) emission that is an extension of the Fermi spectra to higher energies, while in other cases, additional higher-energy spectral components that require a separate emission mechanism may be present. We will present results of broadband spectral modeling using global magnetosphere fields and multiple emission mechanisms that include synchro-curvature (SC) and inverse Compton scattered (ICS) radiation from accelerated particles (primaries) and synchrotron self-Compton (SSC) emission from lower-energy pairs. Our models predict three distinct VHE components: SC from primaries whose high-energy tail can extend to $100 \mathrm{GeV}$, SSC from pairs that can extend to several TeV and ICS from primary particles accelerated in the current sheet, scattering pair synchrotron radiation, that appears beyond $10 \mathrm{TeV}$. Model spectra show a wide range of VHE flux, with detectable pair SSC and primary ICS components expected for Crablike pulsars and some millisecond pulsars but only a primary ICS component for Vela. Our models suggest that H.E.S.S.-II and MAGIC have detected the high-energy tail of the primary SC component that produces the Fermi spectrum in Vela, Geminga and PSR B1706-44. We argue that the ICS component peaking above $10 \mathrm{TeV}$ from Vela has been seen by H.E.S.S. Detection of this emission component from the Crab and other pulsars is possible with HAWC and CTA, and directly measures the maximum particle energy in pulsars.

$37^{\text {th }}$ International Cosmic Ray Conference (ICRC 2021)

July 12th - 23rd, 2021

Online - Berlin, Germany

\footnotetext{
${ }^{*}$ Presenter
} 


\section{Introduction}

The last decade has seen a rapid increase in sensitivity of ground-based Air-Cherenkov telescopes to detect pulsed VHE emission from rotation-powered pulsars above energies of $10 \mathrm{GeV}$. MAGIC first announced the detection of pulsations from the Crab pulsar at energies up to $\sim 25 \mathrm{GeV}$ [1], followed by the VERITAS detection of pulsed photons up to $\sim 400 \mathrm{GeV}$ [2], and finally, MAGIC detected pulsations up to $1.5 \mathrm{TeV}$ [3]. H.E.S.S.-II then detected pulsed emission from the Vela pulsar in the sub-20 GeV to $100 \mathrm{GeV}$ range [4]. H.E.S.S. also reported pulsed emission from this pulsar at a few $\mathrm{TeV}$ (H.E.S.S. Collaboration, in preparation). Most recently, H.E.S.S.-II detected pulsed emission from PSR B1706-44 up to $70 \mathrm{GeV}$ [5]. Finally, MAGIC has detected pulsed emission from the Geminga pulsar between $15 \mathrm{GeV}$ and $75 \mathrm{GeV}$ at a significance of $6.3 \sigma$ [6]. The measured spectra in the sub-100 GeV range for Vela, B1706-44 and Geminga seem to smoothly connect to that measured by Fermi Large Area Telescope (LAT), so it is not clear whether this emission requires a separate spectral component. Also in all three cases, only the second light curve peak is visible, extending the trend of disappearing first peaks with increasing energy observed by Fermi to these higher energies.

We present here some results of our recent broadband modeling of these pulsars, providing possible physical explanations for the observed components as well as predictions for future observations by the existing telescopes and by the anticipated operation of CTA. Recent advances in global pulsar magnetosphere simulations have shown that most pulsars must have a near force-free field with most dissipation and particle acceleration located near the current sheet outside the light cylinder [7, 9]. We therefore use the magnetic field structure of a global force-free magnetosphere for our radiation model, so that we expect the VHE emission components to come primarily from the current sheet. The details of the emission model and a full presentation and discussion of the results are given in [10]. Here, we will highlight a few of the important model features and show some of the modeled spectra and light curves. Finally, prospects for future observations of VHE emission from pulsars will be discussed.

\section{Emission Model}

Details of the broadband radiation model can be found in [10,11] and [12], so we will only provide an overview of the calculation here. We inject two populations of particles at the neutron star surface in a global force-free magnetosphere: "primary" particles (either electrons of positrons) that are accelerated by electric fields parallel to the local magnetic field, $E_{\|}$, and a spectrum of electron-positron pairs created in a polar cap cascade. The pair spectra are computed for each pulsar we model through a separate local simulation [13]. The particles are injected near the rims of the polar caps and their trajectories and dynamics are computed in the force-free geometry. The $E_{\|}$ acceleration is assumed to be constant with a lower value below the light cylinder and higher value in the current sheet. This two-tier $E_{\|}$is guided by global particle-in-cell models that show the highest acceleration in the current sheet starting at the Y-point and a low level of acceleration below the light cylinder [14]. The pairs are not accelerated along their trajectories. All particles radiate synchro-curvature (SC) and inverse-Compton scattering (ICS) radiation. The code loops through all particle trajectories twice, the first time to compute the SC radiation and grid of emissivities 


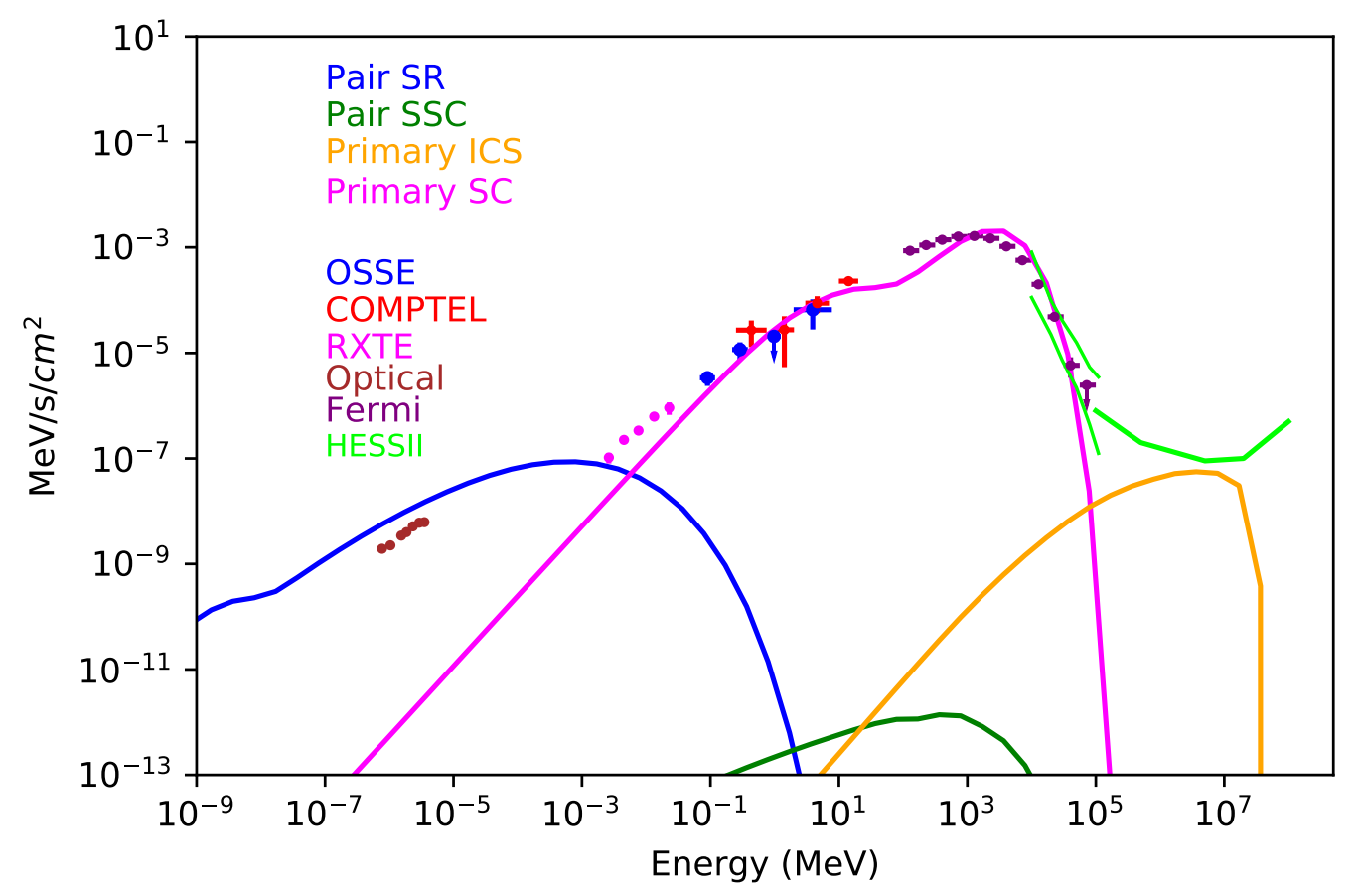

Figure 1: Model spectral energy distribution for the Vela pulsar for inclination angle $\alpha=75^{\circ}$ and viewing angle $\zeta=50^{\circ}$. Data points are from [16] (http://fermi.gsfc.nasa.gov/ssc/data/access/lat/2nd_PSR_catalog/), [17], and [18]. The H.E.S.S.-II detection [4] and high-energy sensitivity are also shown.

and the second time to compute the ICS emission using the SC emissivities from the first loop. Both pairs and primaries are injected with zero pitch angles but can acquire finite pitch angles through resonant absorption of radio photons that are emitted at a specified altitude, $r_{\text {radio }}$, above the neutron star surface. The primaries will therefore radiate a mixture of synchrotron radiation (SR) and curvature radiation (CR) that is captured by treating the radiation as SC [15], with the emission below the light cylinder being mostly SR, becoming mostly CR in the current sheet as particle Lorentz factors exceed $10^{7}$. Since the pairs have relatively low Lorentz factors, their radiation in the first loop is in the SR limit of SC emission.

\section{Results}

The model has six adjustable parameters: magnetic inclination angle, $\alpha$, the accelerating electric field below, $E_{\|}^{\text {low }}$, and above, $E_{\|}^{\text {high }}$, the light cylinder, the primary particle current, $J / J_{\mathrm{GJ}}$, in units of the Goldreich-Julian current density, $J_{\mathrm{GJ}}=\rho_{\mathrm{GJ}} c$, where $\rho_{\mathrm{GJ}}=B \Omega / 2 \pi c$, the pair multiplicity, $M_{+}$, and the radio emission altitude, $r_{\text {radio }}$. For all sources, the $E_{\|}^{\text {low }}, E_{\|}^{\text {high }}$ and $J / J_{\mathrm{GJ}}$ parameters are adjusted so that the SC component matches the hard X-ray to GeV data, particularly the high-energy cutoff in the Fermi SEDs which is especially sensitive to $E_{\|}^{h i g h}$. The hard X-ray SED is most sensitive to the value of $E_{\|}^{\text {low }}$. The $M_{+}$and $r_{\text {radio }}$ are adjusted so that the pair SR matches the observed IR to soft X-ray data. Then the viewing angle, $\zeta$, is chosen to best match the 


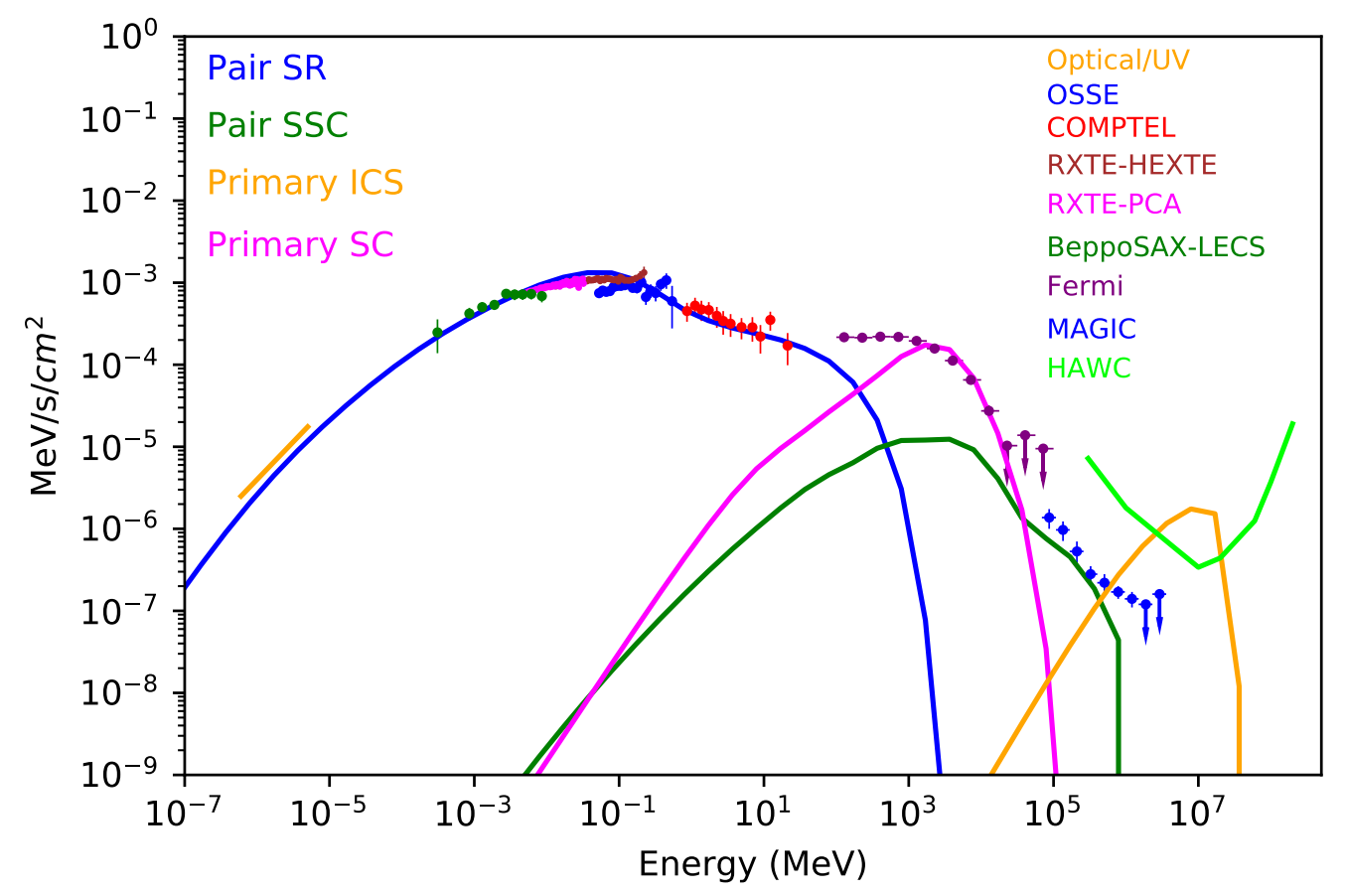

Figure 2: Model spectral energy distribution for the Crab pulsar for inclination angle $\alpha=45^{\circ}, \zeta=66^{\circ}$. Data points are from [20], [16], [3] and [21].

observed Fermi and VHE light curves. After these parameters are adjusted, the ICS component fluxes and SEDs are determined without further parameter adjustments.

Figure 1 shows a model spectral energy distribution (SED) for the Vela pulsar. The result is similar to that shown in [11] but with notable differences. The pair spectrum used in this calculation assumes a non-dipolar field [19] but the same $M_{+}=6 \times 10^{3}$. Since the non-dipolar pair spectrum extends to lower pair energy, the pair SR spectrum also extends to lower photon energy and the IR/Optical flux is higher. Consequentially, this model primary ICS flux is higher by almost an order of magnitude and nearly reaches the H.E.S.S.-II threshold. The primary SC component, whose high-energy tail can account for the H.E.S.S.-II measurement, is the same as shown in [11] since the $E_{\|}^{\text {low }}$ and $E_{\|}^{\text {high }}$ values are the same. Viewing angles in the range $50^{\circ}$ to $70^{\circ}$ make minor differences to the primary ICS component.

The model SED for the Crab pulsar is shown in Figure 2 using a pair energy spectrum that assumes a pure dipole field but with a power-law extension in order to match the COMPTEL soft $\gamma$-ray points. We have assumed a pair multiplicity $M_{+}=3 \times 10^{5}$. This pair spectrum produces a pair SSC spectrum that also better matches the MAGIC VHE points. In our model, the MAGIC spectrum is therefore not an extension of the primary SC spectrum, predicting a possible dip or discontinuity between the Fermi and MAGIC spectra. Our model also predicts a primary ICS component peaking around $10-20 \mathrm{TeV}$ that is potentially detectable by HAWC, although we have included a possibly under-estimated $\gamma-\gamma$ pair attenuation.

In Figure 3 we show a model SED for the Geminga pulsar for $M_{+}=2 \times 10^{4}$ and assuming 


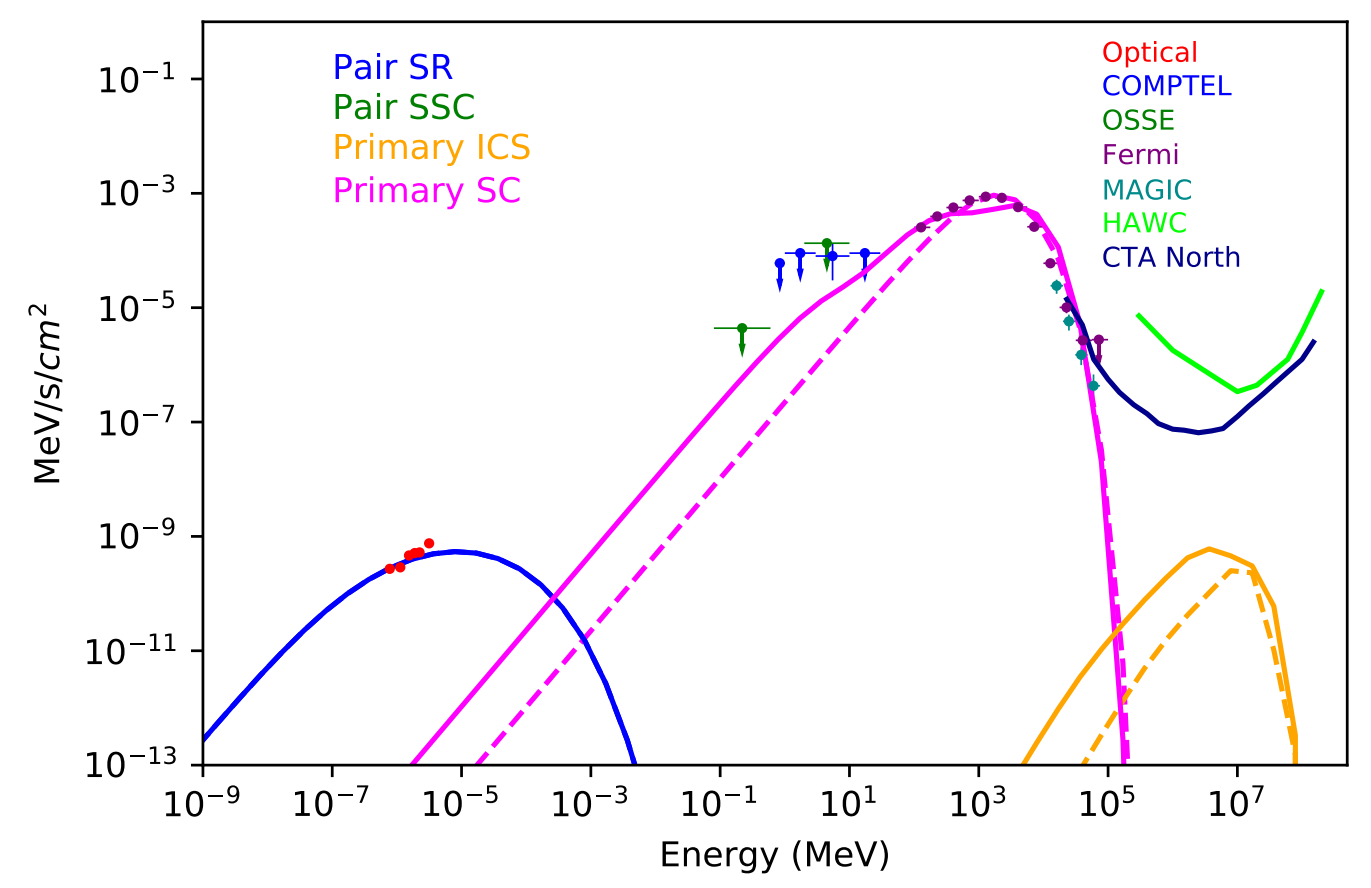

Figure 3: Model spectral energy distribution for the Geminga pulsar for inclination angle $\alpha=75^{\circ}$, viewing angle $\zeta=50^{\circ}$. Data are from [22], [23], [24], [16], and [6].

a single value $E_{\|}^{\text {low }}=E_{\|}^{\text {high }}$ at all radii. Since Geminga does not have detected radio emission, we assume a radio flux of $1000 \mathrm{mJy}$ which can seed the observed optical/UV emission through the pair SR component. A viewing angle of $\zeta \sim 50^{\circ}$ produces a flux of pair SR and shape of primary SC emission that best matches the data. Since the hard X-ray spectrum of Geminga is not well measured, the $E_{\|}^{\text {low }}$ value is presently unconstrained. We see that the MAGIC spectrum that is an extension of the Fermi spectrum is well explained as primary SC emission with no need to invoke any ICS component. Indeed, our predicted ICS emission, both from pairs (whose flux is below the level of the plot) and primaries, is well below any present detection thresholds. Since the computed Geminga pair spectrum is much lower than that of Vela in total multiplicity and in low and high-energy extent, the predicted pair SR spectrum for Geminga has a lower flux and SED energy peak, both by about two orders of magnitude, and a narrower energy range compared with Vela. The light cylinder distance is also three times larger than for Vela, so that the the high-energy particles in the current sheet are farther from the soft photon source making their local density lower. So even though the primary SC flux is comparable to Vela, the lower soft photon density produces a lower primary ICS component

Figure 4 shows our SED model for PSR B1706-44, a Vela-like pulsar, for inclination and viewing angles chosen to match the Fermi light curve which is very different from that of Vela. There is no pulsed optical emission detected for this pulsar, since its distance is much larger than Vela's or Geminga's but there is a soft power-law X-ray detection from [25] which we use to match the flux of the pair SR component. The predicted pair SR spectrum has a higher flux (by two orders 


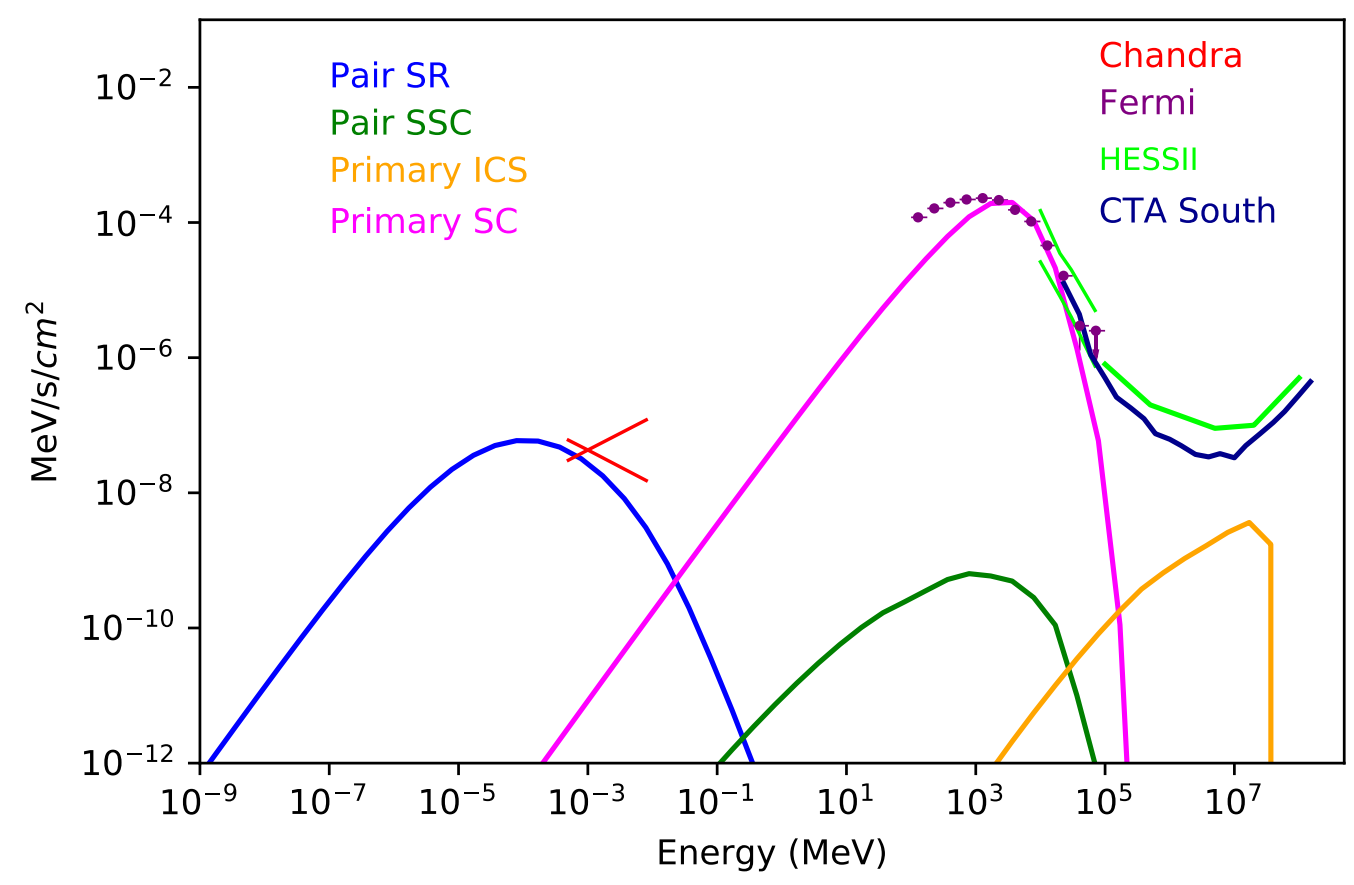

Figure 4: Model spectral energy distribution for PSR B1706-44 for inclination angle $\alpha=45^{\circ}$ and viewing angle $\zeta=53^{\circ}$. Data are from [25], [16] and [5].

of magnitude) and SED peak energy (by a factor of ten) than for Geminga, primarily because its pair spectrum is higher in multiplicity and extends to higher energy. The luminosity of the primary ICS is predicted to be higher than Vela's and it is only its larger distance and relatively lower predicted optical/IR flux that makes the flux level below detection thresholds. However, we predict that the flux of the primary ICS component is a factor of ten above that for Geminga. H.E.S.S.-II has detected pulsed emission up to $70 \mathrm{GeV}$ which we can explain as primary SC emission, as for Geminga.

\section{Conclusions}

Our model of broadband emission from rotation-powered pulsars predicts three different pulsed VHE components. The first is primary SC radiation which has a high-energy tail that extends to around $100 \mathrm{GeV}$. This emission, which can account for the sub-100 GeV detections of Vela and PSR B1706-44 by H.E.S.S.-II and of Geminga by MAGIC, results from radiation of particles highly accelerated in the current sheet with different radiation-reaction limited energies. The particle trajectory radii of curvature vary at different altitudes along the current sheet, producing a range of CR cutoffs that all combine in the caustic peaks. This component should be detectable from pulsars with high Fermi flux. SSC radiation from pairs produces a second VHE component with a broad SED whose peak typically occurs between $1-10 \mathrm{GeV}$ for young pulsars. Although the pair SSC emission is obscured by the primary SC below $100 \mathrm{GeV}$, it is visible up to about $1 \mathrm{TeV}$ 
and may account for the MAGIC measured emission from the Crab. The third predicted VHE component is produced by primary ICS, from the most highly accelerated particles in the current sheet scattering low-energy pair SR. The SED extends from $100 \mathrm{GeV}$ to a sharp cutoff around $30 \mathrm{TeV}$, the maximum energy of accelerated particles. Detection of the high-energy cutoff can therefore measure the maximum particle energy. We predict that the primary ICS component will be detectable from Vela, Crab and possibly other similar pulsars with short periods and high optical/IR flux.

\section{References}

[1] E. Aliu and MAGIC Collaboration, Observation of Pulsed $\gamma$-Rays Above $25 \mathrm{GeV}$ from the Crab Pulsar with MAGIC, Science 322 (Nov., 2008) 1221, [arXiv: 0809. 2998].

[2] E. Aliu and VERITAS Coll., Detection of Pulsed Gamma Rays Above $100 \mathrm{GeV}$ from the Crab Pulsar, Science 334 (Oct., 2011) 69, [arXiv: 1108. 3797].

[3] S. Ansoldi and MAGIC Collaboration, Teraelectronvolt pulsed emission from the Crab Pulsar detected by MAGIC, 585 (Jan., 2016) A133, [arXiv: 1510.07048].

[4] H. Abdalla and HESS Collaboration, First ground-based measurement of sub-20 GeV to 100 GeV $\gamma$-Rays from the Vela pulsar with H.E.S.S. II, 620 (Dec., 2018) A66, [arXiv: 1807.01302].

[5] M. Spir-Jacob, A. Djannati-Ataï, L. Mohrmann, G. Giavitto, B. Khélifi, B. Rudak, C. Venter, and R. Zanin, Detection of sub-100 GeV gamma-ray pulsations from PSR B1706-44 with H.E.S.S, arXiv e-prints (Aug., 2019) arXiv:1908.06464, [arXiv: 1908.06464].

[6] V. A. Acciari and MAGIC Collaboration, Detection of the Geminga pulsar with MAGIC hints at a power-law tail emission beyond $15 \mathrm{GeV}, \mathbf{6 4 3}$ (2020) L14.

[7] C. Kalapotharakos, A. K. Harding, and D. Kazanas, Gamma-Ray Emission in Dissipative Pulsar Magnetospheres: From Theory to Fermi Observations, 793 (Oct., 2014) 97, [arXiv: 1310.3545].

[8] A. A. Philippov and A. Spitkovsky, Ab-initio Pulsar Magnetosphere: Particle Acceleration in Oblique Rotators and High-energy Emission Modeling, 855 (Mar., 2018) 94, [arXiv: 1707.04323].

[9] C. Kalapotharakos, G. Brambilla, A. Timokhin, A. K. Harding, and D. Kazanas, Three-dimensional Kinetic Pulsar Magnetosphere Models: Connecting to Gamma-Ray Observations, 857 (Apr., 2018) 44, [arXiv: 1710.03170].

[10] A. K. Harding, C. Venter, and C. Kalapotharakos, Very-High-Energy Emission From Pulsars, in prep. (2021).

[11] A. K. Harding, C. Kalapotharakos, M. Barnard, and C. Venter, Multi-TeV Emission from the Vela Pulsar, 869 (Dec., 2018) L18, [arXiv: 1811.11157]. 
[12] A. K. Harding and C. Kalapotharakos, Synchrotron Self-Compton Emission from the Crab and Other Pulsars, 811 (Sept., 2015) 63, [arXiv: 1508.06251].

[13] A. N. Timokhin and A. K. Harding, On the Polar Cap Cascade Pair Multiplicity of Young Pulsars, 810 (Sept., 2015) 144, [arXiv: 1504.02194$].$

[14] G. Brambilla, C. Kalapotharakos, A. N. Timokhin, A. K. Harding, and D. Kazanas, Electron-Positron Pair Flow and Current Composition in the Pulsar Magnetosphere, 858 (May, 2018) 81, [arXiv: 1710.03536].

[15] D. F. Torres, Order parameters for the high-energy spectra of pulsars, Nature Astronomy 2 (Feb., 2018) 247-256, [arXiv : 1802 . 04177].

[16] A. A. Abdo and Fermi Collaboration, The Second Fermi Large Area Telescope Catalog of Gamma-Ray Pulsars, 208 (Oct., 2013) 17, [arXiv: 1305.4385].

[17] Y. A. Shibanov, A. B. Koptsevich, J. Sollerman, and P. Lundqvist, The Vela pulsar in the near-infrared, 406 (Aug., 2003) 645-655, [astro-ph/0305273].

[18] A. K. Harding, M. S. Strickman, C. Gwinn, R. Dodson, D. Moffet, and P. McCulloch, The Multicomponent Nature of the Vela Pulsar Nonthermal X-Ray Spectrum, 576 (Sept., 2002) 376-380, [astro-ph/0205183].

[19] A. K. Harding and A. G. Muslimov, Pulsar Pair Cascades in Magnetic Fields with Offset Polar Caps, 743 (Dec., 2011) 181, [arXiv: 1111. 1668].

[20] L. Kuiper, W. Hermsen, G. Cusumano, R. Diehl, V. Schönfelder, A. Strong, K. Bennett, and M. L. McConnell, The Crab pulsar in the 0.75-30 MeV range as seen by CGRO COMPTEL. A coherent high-energy picture from soft $X$-rays up to high-energy gamma-rays, 378 (Nov., 2001) 918-935, [astro-ph/0109200].

[21] J. Sollerman, J. Selsing, P. M. Vreeswijk, P. Lundqvist, and A. Nyholm, The optical and NIR spectrum of the Crab pulsar with X-shooter, 629 (Sept., 2019) A140, [arXiv: 1906.09878].

[22] O. Y. Kargaltsev, G. G. Pavlov, V. E. Zavlin, and R. W. Romani, Ultraviolet, X-Ray, and Optical Radiation from the Geminga Pulsar, 625 (May, 2005) 307-323, [astro-ph/0502076].

[23] Y. A. Shibanov, S. V. Zharikov, V. N. Komarova, N. Kawai, Y. Urata, A. B. Koptsevich, V. V. Sokolov, S. Shibata, and N. Shibazaki, Subaru optical observations of the two middle-aged pulsars PSR B0656+14 and Geminga, 448 (Mar., 2006) 313-326, [astro-ph/0511311].

[24] L. Kuiper, W. Hermsen, K. Bennett, A. Connors, R. Much, J. Ryan, V. Schoenfelder, and A. Strong, Search in the COMPTEL data for MeV-emission from Geminga., 120 (Nov., 1996) 73-76.

[25] E. V. Gotthelf, J. P. Halpern, and R. Dodson, Detection of Pulsed X-Ray Emission from PSR B1706-44, 567 (Mar., 2002) L125-L128, [astro-ph/0201161]. 